\title{
Supercover Model and Digital Straight Line Recognition on Irregular Isothetic Grids
}

\author{
David Coeurjolly \\ Laboratoire LIRIS - CNRS FRE 2672, \\ Universit Claude Bernard Lyon1, \\ 43 Bd du 11 novembre 1918, \\ Villeurbanne, France \\ dcoeurjo@liris.cnrs.fr
}

\begin{abstract}
On the classical discrete grid, the analysis of digital straight lines (DSL for short) has been intensively studied for nearly half a century. In this article, we are interested in a discrete geometry on irregular grids. More precisely, our goal is to define geometrical properties on irregular isothetic grids that are tilings of the Euclidean plane with different sized axis parallel rectangles.
\end{abstract}

\section{Introduction}

When a straight line is digitized on a square grid, we obtain a sequence of grid points defining a digital straight-line segment. This computer representation of such a simple Euclidean object has drawn considerable attention in many applications (drawing [3, shape characterization 13, 14, 17, 7, ...). The structure of DSL is now well known and links have been illustrated between DSL and objects from number theory or theory of words (see Rosenfeld and Klette [26] for a survey on digital straightness). Beyond this characterization, an important task in computer vision consists in the recognition of DSL segments. More precisely, given a set of pixels, we have to decide if there exists a DSL segment that contains the given pixels. Many efficient algorithms exist to implement such a recognition process 15, 18, 11, 4. Based on a digital straight line recognition algorithm, we can also define a segmentation process that decomposes a discrete curve into maximal DSL segments.

In this article, we are interested in defining a geometry on irregular isothetic grids. More precisely, we consider grids defined by a tiling of the plane using axis parallel rectangles. Such a grid model includes, for example, the classical discrete grid, the elongated grids [27] and the quadtree based grids [16]. In the following sections, we focus a general supercover digitization model on the irregular isothetic grids which is consistent with the classical one if the discrete space is considered. A previous work can be found in [8] in which irregular grids with squares are considered. In this model, the chosen digitization scheme is the naive model and it suffers from some inconsistencies. For example, the digitization of an Euclidean straight line may be a disconnected set of pixels. In the following, 
we generalize the model to irregular tilings of rectangles with an appropriate digitization model.

Many applications may benefit from these developments. For example, we can cite the analysis of quadtree compressed shape, or the use of geometrical properties in objects represented by interval or affine arithmetics [21, 22, 10, 5]. In this last example, we talk about data driven grids.

Section 2 presents more formal definitions of the irregular grids which allow to define the supercover model in Section [3. Then, we present the definition and the recognition algorithm of digital straight lines in these grids (Section 4). Experiments and results are shown in Section 5. Finally, we briefly illustrate the application of the irregular model in interval arithmetic (Section 6).

\section{Definitions}

First of all, we define an irregular isothetic grid, denoted $\mathbb{I}$, as a tiling of the plane with isothetic rectangles. In this framework, the rectangles have not necessarily the same size but we can notice that the classical digital space is a particular irregular isothetic grid. In that case, all squares are centered in $\mathbb{Z}^{2}$ points and have a border size equal to 1. Figure 1 illustrates some examples of irregular isothetic grids.

In the following, a rectangle of an isothetic grid is called a pixel. Each pixel $P$ is defined by its center $\left(x_{P}, y_{P}\right) \in \mathbb{R}^{2}$ and a size $\left(l_{P}^{x}, l_{P}^{y}\right) \in \mathbb{R}^{2}$. Before we introduce objects and straight lines in such grids, we need adjacency relations between pixels.
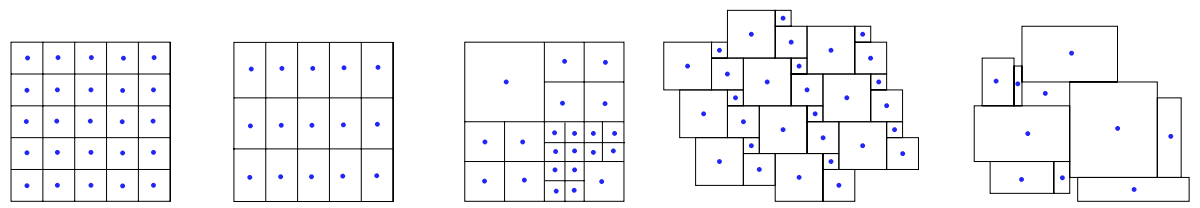

Fig. 1. Examples of irregular isothetic grids: (from left to right) the classical discrete grid $\left(\left(x_{P}, y_{P}\right) \in \mathbb{Z}^{2}\right.$ and $\left.l_{P}^{x}=l_{P}^{y}=1\right)$, an elongated grid $\left(l_{P}^{x}=\lambda, l_{P}^{y}=\mu\right.$ and $\left(x_{P}, y_{P}\right)=(\lambda i, \mu j)$ with $\left.(i, j) \in \mathbb{Z}^{2}\right)$, a quadtree decomposition (for a cell of level $k,\left(x_{P}, y_{P}\right)=\left(\frac{m}{2^{k}}, \frac{n}{2^{k}}\right)$ and $l_{P}^{x}=l_{P}^{y}=\frac{1}{2^{k-1}}$ for some $\left.m, n \in \mathbb{Z}\right)$; a unilateral and equitransitive tiling by squares: the size of the biggest square is equal to the sum of the two other square sizes; finally a general irregular isothetic grid

Definition 1 (ve-adjacency, e-adjacency). Let $P$ and $Q$ be two pixels. $P$ and $Q$ are ve-adjacent if:

$$
\left|x_{P}-x_{Q}\right|=\frac{l_{P}^{x}+l_{Q}^{x}}{2} \text { and }\left|y_{P}-y_{Q}\right| \leq \frac{l_{P}^{y}+l_{Q}^{y}}{2},
$$

or

$$
\left|y_{P}-y_{Q}\right|=\frac{l_{P}^{y}+l_{Q}^{y}}{2} \text { and }\left|x_{P}-x_{Q}\right| \leq \frac{l_{P}^{x}+l_{Q}^{x}}{2}
$$


$P$ and $Q$ are e-adjacent if we consider an exclusive "or" and strict inequalities in the above ve-adjacent definition.

In the following definitions, we use the notation $k$-adjacency in order to express either the ve-adjacency or the e-adjacency. Using these adjacency definitions, several basic objects can be defined:

Definition 2 ( $k$-path). Let us consider a set of pixels $\mathcal{E}=\left\{P_{i}, i \in\{1, \ldots, n\}\right\}$ and a relation of $k$-adjacency. $\mathcal{E}$ is a $k$-path if and only if for each element $P_{i}$ of $\mathcal{E}, P_{i}$ is $k$-adjacent to $P_{i-1}$.

Definition 3 ( $k$-object). Let $\mathcal{E}$ be a set of pixels, $\mathcal{E}$ is a $k$-object if and only if for each couple of pixels $(P, Q)$ belonging to $\mathcal{E} \times \mathcal{E}$, there exists a $k$-path between $P$ and $Q$ in $\mathcal{E}$.

Definition 4 (k-arc). Let $\mathcal{E}$ be a set of pixels, $\mathcal{E}$ is a $k$-arc if and only if for each the element of $\mathcal{E}=\left\{P_{i}, i \in\{1, \ldots, n\}\right\}, P_{i}$ has exactly two $k$-adjacent pixels, except $P_{1}$ and $P_{n}$ which are called the extremities of the $k$-arc.

Definition 5 (k-curve). Let $\mathcal{E}$ be a set of pixels, $\mathcal{E}$ is a $k$-curve if and only if $\mathcal{E}$ is a $k$-arc and $P_{1}=P_{n}$.

If we consider pixels such that $l_{P}^{x}=l_{P}^{y}=1$ and $\left(x_{P}, y_{P}\right) \in \mathbb{Z}^{2}$ (i.e. a $2 \mathrm{D}$ digital space), all these definitions coincide with the classical ones 24,25]. More precisely, the $v e$-adjacency (resp. $e$-adjacency) is exactly the 8-adjacency (resp. the 4-adjacency).

In the following, we only consider geometrical properties of such objects. A complete topological analysis of $k$-curves and $k$-objects is not addressed here.

\section{Supercover Model on the Irregular Isothetic Grids}

Before defining the digital straight lines on the irregular isothetic grids, we have to consider a digitization model. In the following, we choose to extend the supercover model. This model was first introduced by Cohen-Or and Kaufman in [9] on the classical discrete grid and then widely used since it provides an analytical characterization of basic supercover objects (e.g. lines, planes, 3D polygons, ...) [2,1].

Definition 6 (Supercover on irregular isothetic grids). Let $F$ be an Euclidean object in $\mathbb{R}^{2}$. The supercover $\mathbb{S}(F)$ is defined on an irregular isothetic grid II by:

$$
\begin{aligned}
\mathbb{S}(F)=\{P \in \mathbb{I} & \left.\mid \quad \mathbb{B}^{\infty}(P) \cap F \neq \emptyset\right\} \\
& =\left\{P \in \mathbb{I} \quad|\quad \exists(x, y) \in F,| x_{P}-x \mid \leq \frac{l_{P}^{x}}{2} \text { and }\left|y_{P}-y\right| \leq \frac{l_{P}^{y}}{2}\right\}
\end{aligned}
$$



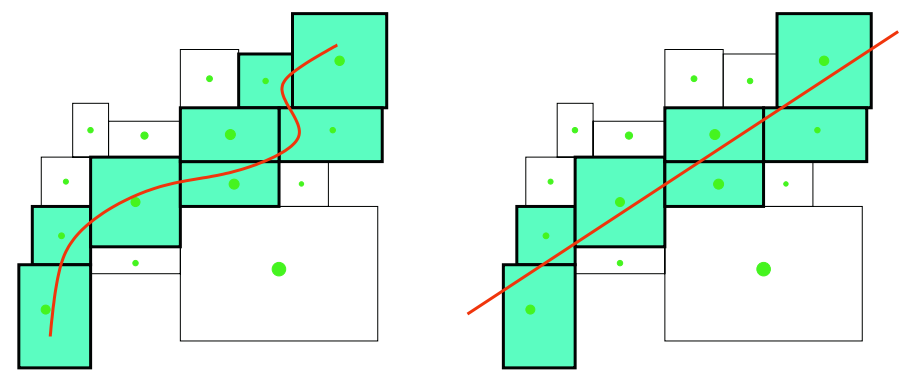

Fig. 2. Illustration of the supercover digitization of a curve (left) and of a straight line (right)

where $\mathbb{B}^{\infty}(P)$ is the rectangle centered in $\left(x_{P}, y_{P}\right)$ of size $\left(l_{P}^{x}, l_{P}^{y}\right)$ (if $l_{P}^{x}=l_{P}^{y}$, $\mathbb{B}^{\infty}(P)$ is the ball centered in $\left(x_{P}, y_{P}\right)$ of size $l_{P}^{x}$ for the $L_{\infty}$ norm).

This model has got several properties:

Proposition 1. Let $F, G$ be Euclidean objects in $\mathbb{R}^{2}, \alpha \in \mathbb{R}^{2}$ and an $\mathbb{I}$-grid, we have:

$$
\begin{aligned}
& \mathbb{S}(F \cup G)=\mathbb{S}(F) \cup \mathbb{S}(G), \\
& \mathbb{S}(F)=\bigcup_{\alpha \in F} \mathbb{S}(\alpha), \\
& \mathbb{S}(F \cap G) \subseteq \mathbb{S}(F) \cap \mathbb{S}(G), \\
& \text { if } F \subseteq G \text { then } \mathbb{S}(F) \subseteq \mathbb{S}(G) .
\end{aligned}
$$

Proof. All these statements can be easily proved by definition of the supercover model. For example, we prove the first one as follows:

$$
\begin{aligned}
\mathbb{S}(F \cup G) & =\left\{p \in \mathbb{I} \quad \mid \quad \mathbb{B}^{\infty}(P) \cap(F \cup G) \neq \emptyset\right\} \\
& =\left\{p \in \mathbb{I} \quad \mid \quad\left(\mathbb{B}^{\infty}(P) \cap F\right) \cup\left(\mathbb{B}^{\infty}(P) \cap G\right) \neq \emptyset\right\} \\
& =\mathbb{S}(F) \cup \mathbb{S}(G)
\end{aligned}
$$

Figure 2 illustrates some examples of the supercover digitization of Euclidean objects.

If $\mathbb{I}$ is the classical digital space (i.e. $\left(x_{P}, y_{P}\right) \in \mathbb{Z}^{2}$ and $\left.l_{P}^{x}=l_{P}^{y}=1\right)$, many links exist between the supercover of an Euclidean straight line and classical digital straight line definitions 1,26 . Since we have not any assumption on the irregular grid, no strong topological property can be stated on the supercover of an Euclidean straight line.

Proposition 2. Let $l$ be an Euclidean straight line and a $\mathbb{I}$-grid, the $\mathbb{S}(l)$ is a single ve-object.

Proof. The proof is direct since $\mathbb{I}$ is a tiling of the plane with closed pattern. The digitization of $l$ is necessarily a connected set of pixels. 


\section{Digital Straight Line Definition and Recognition}

\subsection{Definitions}

Definition 7 (Irregular Isothetic Digital Straight Line). Let $S$ be a set of pixels in $\mathbb{I}, S$ is called a piece of irregular digital straight line (IDSL for short) iff there exists an Euclidean straight line l such that:

$$
S \subseteq \mathbb{S}(l)
$$

In other words, $S$ is a piece of IDSL iff there exists l such that:

$$
\forall P \in S, \quad \mathbb{B}^{\infty}(P) \cap l \neq \emptyset .
$$

To detect if $\mathbb{B}^{\infty}(P) \cap l$ is empty or not, we use the notations presented in Figure 3. Hence, $\mathbb{B}^{\infty}(P) \cap l$ is not empty iff $l$ crosses either (or both) the diagonals $d_{1}$ or $d_{2}$ of $P$.

Without loss of generality, we suppose that $l$ is given by $y=\alpha x+\beta$ with $(\alpha, \beta) \in \mathbb{R}^{2}$ (an appropriate treatment can be design to handle the straight lines $x=k$ with $k \in \mathbb{R}$ ). To solve the recognition problem, we use the following statement:

$$
\begin{aligned}
\mathbb{B}^{\infty}(P) \cap l \neq \emptyset \quad & \quad l \cap d_{1} \neq \emptyset \text { and } \alpha \geq 0 \\
& \text { or } \quad l \cap d_{2} \neq \emptyset \text { and } \alpha<0
\end{aligned}
$$

During a recognition process, it is convenient to consider the set of Euclidean straight lines whose digitization contains the set of pixels $S$ : if such a set is empty, we can conclude that $S$ is not a discrete straight line segment. In the literature, the set of Euclidean straight lines whose digitization contains $S$ is called the preimage of $S$. Many works have been done concerning the preimage analysis in the classical discrete grid [12, 18, 19].

We first consider Equation (9): given the pixel $P$, the straight line containing $d_{1}$ is

$$
y=-\frac{l_{P}^{y}}{l_{P}^{x}} \cdot x+y_{P}+\frac{l_{P}^{y}}{l_{P}^{x}} \cdot x_{P} .
$$

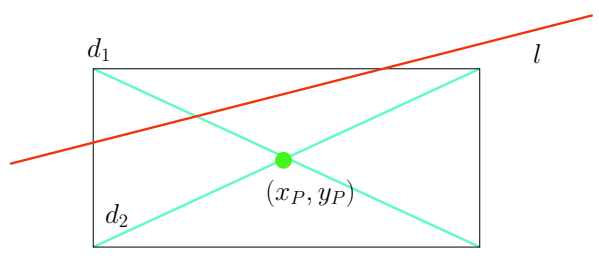

Fig. 3. Notations used to detect if the pixel of center $\left(x_{P}, y_{P}\right)$ belongs to the supercover of a straight line $l\left(d_{1}\right.$ and $d_{2}$ are the diagonals of the rectangle $\left.P\right)$ 
If we compute the intersection point between this straight line and $l$, the abscissa $x$ is given by:

$$
x \cdot\left(\alpha+\frac{l_{P}^{y}}{l_{P}^{x}}\right)=y_{P}+\frac{l_{P}^{y}}{l_{P}^{x}} \cdot x_{P}-\beta .
$$

Hence, to ensure that $l$ intersects $d_{1}, x$ must be such that:

$$
x_{P}-\frac{l_{P}^{x}}{2} \leq x \leq x_{P}+\frac{l_{P}^{x}}{2}
$$

Since $\alpha \geq 0$, we have:

$$
\left(x_{P}-\frac{l_{P}^{x}}{2}\right) \cdot\left(\alpha+\frac{l_{P}^{y}}{l_{P}^{x}}\right) \leq y_{P}+\frac{l_{P}^{y}}{l_{P}^{x}} \cdot x_{P}-\beta \leq\left(x_{P}+\frac{l_{P}^{x}}{2}\right) \cdot\left(\alpha+\frac{l_{P}^{y}}{l_{P}^{x}}\right)
$$

Finally, the condition given in Equation (9) can be represented by the two following inequalities in the $(\alpha, \beta)$-parameter space:

$$
\mathcal{E}^{+}(P)=\left\{\begin{array}{l}
\alpha\left(x_{P}-\frac{l_{P}^{x}}{2}\right)+\beta-y_{P}-\frac{l_{P}^{y}}{2} \leq 0 \\
\alpha\left(x_{P}+\frac{l_{P}^{x}}{2}\right)+\beta-y_{P}+\frac{l_{P}^{y}}{2} \geq 0
\end{array} .\right.
$$

If we consider Equation (10) and using similar arguments, we obtain the following inequalities:

$$
\mathcal{E}^{-}(P)=\left\{\begin{array}{l}
\alpha\left(x_{P}-\frac{l_{P}^{x}}{2}\right)+\beta-y_{P}+\frac{l_{P}^{y}}{2} \geq 0 \\
\alpha\left(x_{P}+\frac{l_{P}^{x}}{2}\right)+\beta-y_{P}-\frac{l_{P}^{y}}{2} \leq 0
\end{array} .\right.
$$

$\mathcal{E}^{+}(P)$ is defined for $\alpha \geq 0$ and $\mathcal{E}^{-}(P)$ for $\alpha<0$. We can now define the preimages of a piece of IDSL:

Definition 8 (Preimages of an IDSL). Let $S$ be a piece of IDSL, the two preimages $\mathcal{P}^{+}$and $\mathcal{P}^{-}$of $S$ are given by:

$$
\begin{aligned}
& \mathcal{P}^{+}(S)=\bigcap_{P \in S} \mathcal{E}^{+}(P), \\
& \mathcal{P}^{-}(S)=\bigcap_{P \in S} \mathcal{E}^{-}(P) .
\end{aligned}
$$

Hence, the recognition process can be described as follows:

Proposition 3. Let $S$ be a set of pixels in a I-grid. $S$ is a piece of IDSL iff $\mathcal{P}^{+}(S) \neq \emptyset$ or $\mathcal{P}^{-}(S) \neq \emptyset$. 


\subsection{Recognition}

Using Proposition 3, the recognition on an IDSL leads to a linear programming problem: we have to decide whether a linear inequality system has a solution or not. More precisely, two different classes of algorithms exist: the IDSL identification algorithms which decide if $S$ is an IDSL or not, and the IDSL recognition algorithms which return the complete preimages (maybe empty) of the recognized IDSL.

Given a linear inequality system, several algorithms can be found to test if a solution exists, i.e. to identify an IDSL. For example, the Meggido's algorithm [20] can decide if a solution exists in $O(n)$ time if $n$ is the number of linear constraints. An incremental version of this algorithm with the same computational cost can also be found [6].

If we need a complete description of the feasible region, i.e. a recognition of the IDSL, Preparata and Shamos [23] proposed an optimal $O(n \log n)$ time online algorithm. This algorithm can easily be implemented since basic computational geometry tools are used (convex hull and dual-space transform).

In Algorithm 1, we present the simple IDSL segmentation algorithm of a $v e-$ arc. The preimage update in lines 6 and 7 can be performed using either an identification or a recognition algorithm.

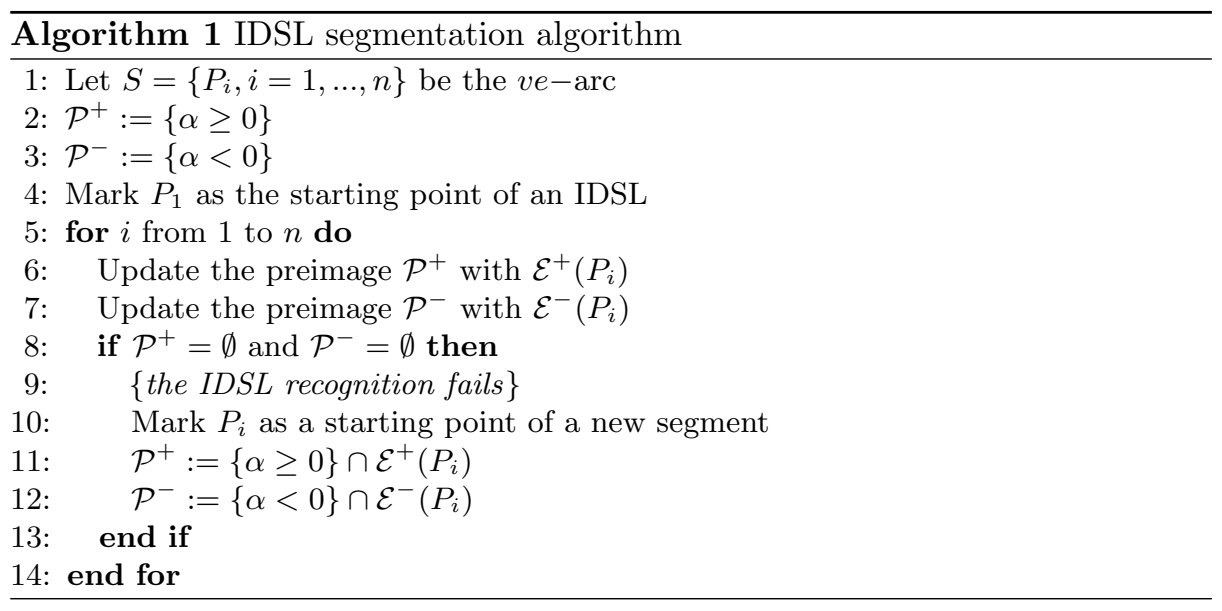

If we consider the classical discrete model $\left(\right.$ i.e. $\left(x_{P}, y_{P}\right) \in \mathbb{Z}^{2}$ and $l_{P}^{x}=l_{P}^{y}=$ 1), Algorithm 1 implements a recognition of classical supercover digital straight lines.

\section{$5 \quad$ Experiments and Results}

In our experiments we have implemented Algorithm 11in MAPLE using the builtin linear programming procedures. First, Figure 4 illustrates the recognition of 


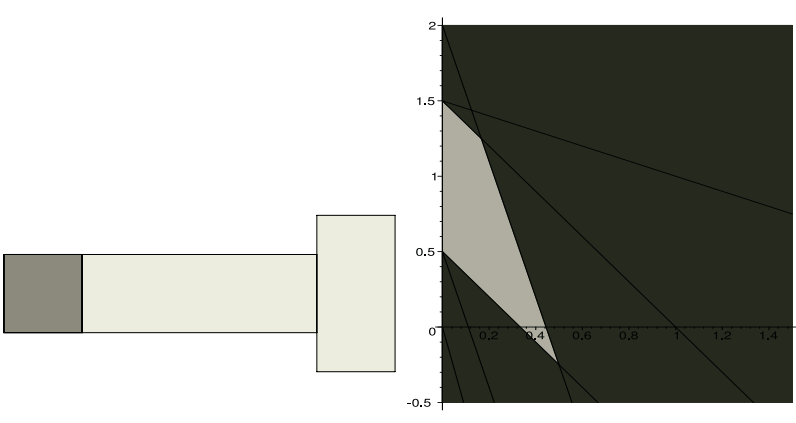

(a) (b)

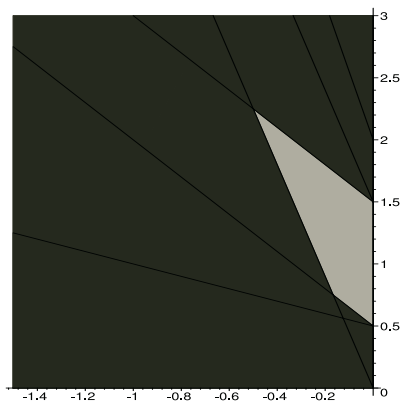

(c)

Fig. 4. Illustration of the preimages associated to a piece of IDSL: $(a)$ a sequence of pixels with $\left(x_{P}, y_{P}, l_{P}^{x}, l_{P}^{y}\right)$ in $\{(1,1,1,1),(3,1,3,1),(5,1,1,2)\},(b)$ its preimage $P^{+}$ and $(c)$, its preimage $P^{-}$in the $(\alpha, \beta)$-parameter space

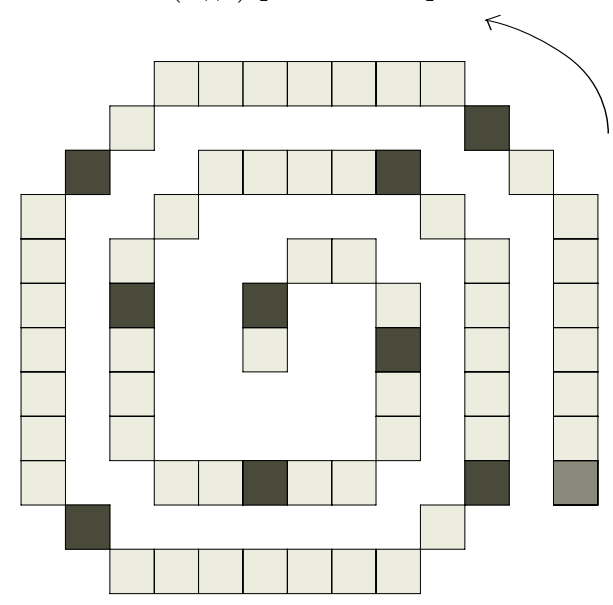

Fig. 5. Segmentation of a classical 8-connected curve into IDSL

a simple piece of IDSL with its associated preimages. Each point $\left(\alpha_{0}, \beta_{0}\right)$ in the preimages $P^{+}$or $P^{-}$(gray areas in Figure $4 .(b-c)$ ), is an Euclidean straight line whose supercover contains the pixels in Figure 4 ( $(a)$.

In Figure 5, we illustrate an IDSL segmentation of a classical discrete curve. The light gray pixel is the starting point of the algorithm and dark gray pixels represent the starting points of the IDSL segments. Note that since the Euclidean straight lines with equations $x=k$ (with $k \in \mathbb{R}$ ) are excluded from the preimages, the IDSL recognition of the left vertical part of the curve differs from the IDSL recognition of the horizontal ones which goes one pixel further.

Figure 6 presents the result on a sequence of pixels defined as the border pixels of a quadtree representation of a binary shape (Figure 6- $(a)$ ). 


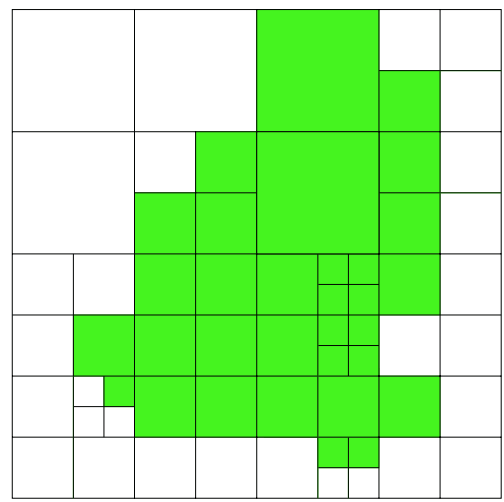

(a)

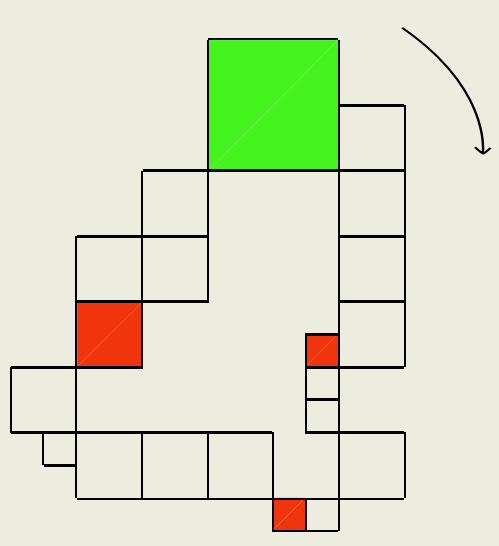

(b)

Fig. 6. (a) Quadtree decomposition of a binary object and (b), the segmentation of its boundary pixels into IDSL

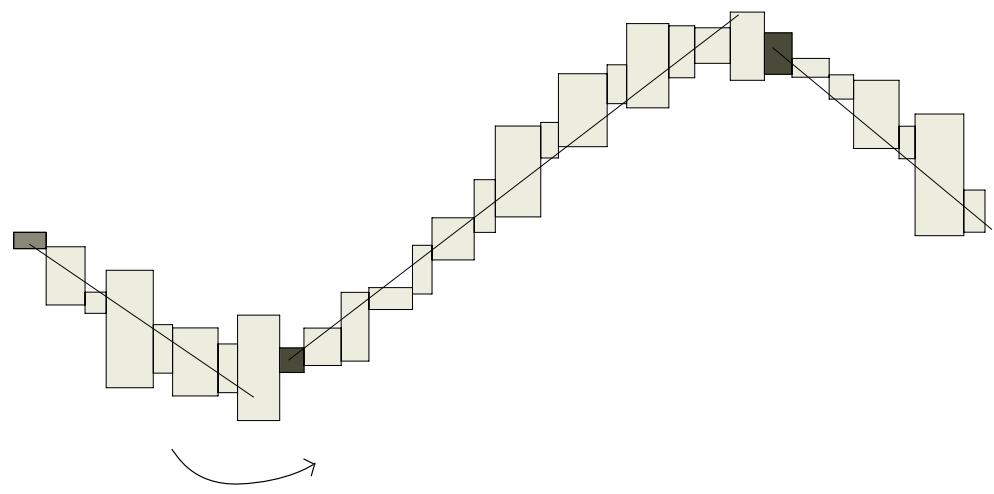

Fig. 7. Illustration of the segmentation algorithm on a general irregular curve. The Euclidean straight lines are manually extracted from the preimages associated to each IDSL segment

Finally, Figure 7 shows the segmentation result on a general ve-curve. In this illustration, we have superposed to the curve an Euclidean straight line per IDSL segment manually extracted from the preimages. 

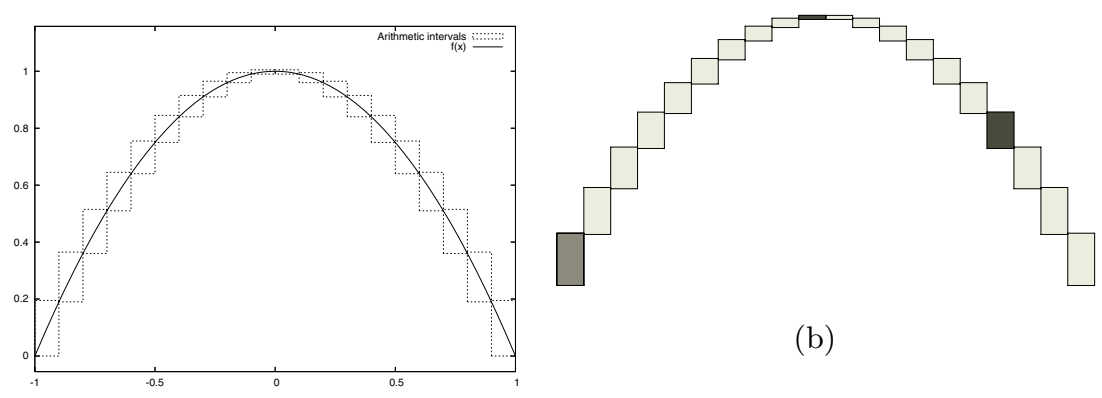

(b)

(a)

Fig. 8. The approximation of the function $f(x)$ using the interval arithmetic $(a)$ and the result of the IDSL segmentation algorithm on these intervals

\section{An Application to Interval Arithmetic Analysis}

In this section, we briefly present an application for which the irregular isothetic model has been developed: the analysis of interval arithmetic objects. The interval arithmetic is a widely used range based model for numerical computation where each quantity $x$ is represented by an interval $\bar{x}$ of floating point numbers [21, 22, 10, 5]. On those intervals, arithmetical operations are defined in such way that each resulting interval $\bar{x}$ is guaranteed to contain the unknown value corresponding to the real $x$ quantity.

Briefly, an interval $\bar{x}$ is represented by $[\bar{x} . l o, \bar{x} . h i]$ and the basic operations are given by:

$$
\begin{aligned}
\bar{x}+\bar{y} & =[\bar{x} . l o+\bar{y} . l o, \bar{x} . h i+\bar{y} . h i] \\
\bar{x}-\bar{y} & =[\bar{x} . l o-\bar{y} \cdot h i, \bar{x} . h i-\bar{y} . l o] \\
\bar{x} \cdot \bar{y} & =[\min (\bar{x} . l o \cdot \bar{y} . l o, \bar{x} . l o \cdot \bar{y} . h i, \bar{x} . h i \cdot \bar{y} . l o, \bar{x} . h i \cdot \bar{y} . h i), \\
& \max (\bar{x} . l o \cdot \bar{y} . l o, \bar{x} . l o \cdot \bar{y} . h i, \bar{x} . h i \cdot \bar{y} . l o, \bar{x} . h i \cdot \bar{y} . h i)]
\end{aligned}
$$

Many other operations can be designed in an interval arithmetic form. In Figure 8 - $(a)$, we have the approximation using interval arithmetic representation of the function $f(\bar{x})=-(\bar{x}-1) \cdot(\bar{x}+1)$ where the intervals $\bar{x}$ are given by a uniform subdivision of $[-1,1]$ into 20 subintervals. Each rectangle in Figure 8 - $(a)$ is given by $[\bar{x} . l o, \bar{x} \cdot h i] \times[f(\bar{x}) \cdot l o, f(\bar{x}) \cdot h i]$. By construction of the intervals and since each operation guarantees that the real function belongs to each interval, we can prove that such an interval arithmetic object is a ve-curve. Hence, the IDSL segmentation algorithm can be applied to obtain a polygonal approximation of the intervals (see Figure $8(b)$ ). In that case, the entire grid $\mathbb{I}$ is unknown, only a subset of it is given. Furthermore, since the set of isothetic irregular pixels are defined by $f(\bar{x})$, we talk about a data driven grid model.

The use of IDSL on objects given by intervals permits to have a first geometrical analysis of the unknown underlying Euclidean curve. 


\section{Conclusion}

In this article, we have presented a global digitization framework on irregular isothetic grids: the supercover model. Based on this digitization scheme, we have defined the digital straight lines in such grids and algorithmic solutions to solve the recognition and segmentation problem. All these developments allow us to characterize and analyze border of quadtree objects for example. Note that all the proposed definitions and algorithms match perfectly with the classical ones in the particular case of the discrete model. We have also illustrated the interest of such a model to analyze object boundaries of quadtree encoded shapes and in the interval arithmetic field.

Future works can be decomposed into two main problems : first the topological and arithmetical analysis of IDSL when a small class of irregular grids is considered (e.g. the quadtree based model). Then, the use of these discrete geometry tools in the interval analysis field.

\section{References}

1. E. Andrès. Modélisation analytique discrète d'objets géométriques. Master's thesis, Laboratoire IRCOM-SIC, Université de Poitiers, 2000.

2. E. Andrès, P. Nehlig, and J. Françon. Tunnel-free supercover 3D polygons and polyhedra. Computer Graphics Forum, 16(3):C3-C13, September 1997.

3. J. Bresenham. An incremental algorithm for digital plotting. In Proc. ACM Natl. Conf., 1963.

4. V. E. Brimkov and S. S. Dantchev. Digital hyperplane recognition in arbitrary fixed dimension. Technical report, CITR-TR-154 Center for Image Technology and Robotics, University of Auckland, New Zealand, 2004.

5. K. Bühler. Linear interval estimations for parametric objects: Theory and application. pages $522-531$.

6. L. Buzer. An incremental linear algorithm for digital line and plane recognition using a linear incremental feasibility problem. In 10th International Conference on Discrete Geometry for Computer Imagery, number 2301 in LNCS, pages 372-381. Springer, 2002.

7. D. Coeurjolly and R. Klette. A comparative evaluation of length estimators of digital curves. IEEE Transactions on Pattern Analysis and Machine Intelligence, 26(2):252-258, feb 2004.

8. D. Coeurjolly and L. Tougne. Digital straight line recognition on heterogeneous grids. In SPIE Vision Geometry XII, volume 5300, pages 108-116, san Jose, USA, 2004.

9. D. Cohen-Or and A. Kaufman. Fundamentals of surface voxelization. Graphical models and image processing: GMIP, 57(6):453-461, November 1995.

10. L.H. de Figueiredo and J. Stolfi. Self-validated

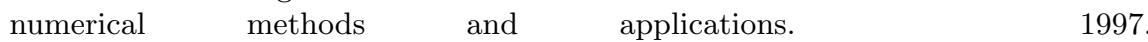
http://www.dcc.unicamp.br/〜stolfi/EXPORT/projects/affine-arith/.

11. I. Debled and J.P. Reveillès. A linear algorithm for segmentation of digital curves. In Third International Workshop on parallel Image Analysis, June 1994.

12. L. Dorst and A. W. M. Smeulders. Discrete representation of straight lines. IEEE Transactions on Pattern Analysis and Machine Intelligence, 6:450-463, 1984. 
13. L. Dorst and A. W. M. Smeulders. Length estimators for digitized contours. Computer Vision, Graphics, and Image Processing, 40(3):311-333, December 1987.

14. L. Dorst and A. W. M. Smeulders. Discrete straight line segments: Parameters, primitives and properties. In R. Melter, P. Bhattacharya, and A. Rosenfeld, editors, Vision Geometry, series Contemporary Mathematics, volume 119, pages 45-62. American Mathematical Society, 1991.

15. A. Hübler E. Creutzburg and V. Wedler. Decomposition of digital arcs and contours into a minimal number of digital straight line segments. In Proc. 6th Intl. Conf. on Pattern Recognition, page 1218, 1982.

16. R. A. Finkel and J. L. Bentley. Quad trees: a dsta structure for retrieval on composite key. Acta Informatica, 4(1):1-9, 1974.

17. R. Klette and B. Yip. The length of digital curves. Machine Graphics 8 S Vision, 9:673-703, 2000. extended version of: R. Klette, V. V. Kovalevsky, B. Yip. Length estimation of digital curves. In Proc. Vision Geometry VIII, SPIE 3811, pages $117-129$.

18. M. Lindenbaum and A. M. Bruckstein. On recursive, $\mathrm{o}(\mathrm{n})$ partitioning of a digitized curve into digital straigth segments. IEEE Transactions on Pattern Analysis and Machine Intelligence, 15(9):949-953, september 1993.

19. M. D. McIlroy. A note on discrete representation of lines. ATEST Technical Journal, 64(2):481-490, February 1985.

20. N. Meggido. Linear programming in linear time when the dimension is fixed. Journal of the ACM, 31(1):114-127, 1984.

21. Ramon E. Moore. Interval Analysis. Prentice-Hall, Englewood Cliffs, N.J., 1966.

22. Ramon E. Moore. Methods and Applications of Interval Arithmetic. Studies in Applied Mathematics. SIAM, Philadelphia, 1979.

23. F. P. Preparata and M. I. Shamos. Computational Geometry : An Introduction. Springer-Verlag, 1985.

24. A. Rosenfeld. Connectivity in digital pictures. Journal of the ACM, 17(1):146-160, January 1970.

25. A. Rosenfeld. Digital straight lines segments. IEEE Transactions on Computers, pages 1264-1369, 1974.

26. A. Rosenfeld and R. Klette. Digital straightness. In S. Fourey, G.T. Herman, and T.Y. Kong, editors, International Workshop on Combinatorial Image Analysis, volume 46 of Electronic Notes in Theoretical Computer Science, Temple University, Philadelphia, U.S.A., August 2001. Elsevier Science Publishers.

27. I.-M. Sintorn and G. Borgefors. Weighted distance transforms for images using elongated voxel grids. In 10th International Conference on Discrete Geometry for Computer Imagery, number 2301 in LNCS, pages 244-254. Springer, 2002. 